\title{
Diagnostic Biomedical Optics and Effect of Nano-Scale Contrast Agents
}

\author{
Murukeshan Vadakke Matham \\ Center for Optical and Laser Engineering \\ Nanyang Technological University \\ Singapore
}

High-resolution diagnostic biomedical optics is an interdisciplinary branch of science and technology, which uses optics for improving the basic understanding of biological processes to enhance the diagnostic efficiency thereby enabling efficient treatment of human diseases. In most of the cases, conventional types of medical imaging may not be able to detect subtle changes occurring in tissues easily. Each imaging modality has its own advantages and limitations and one cannot fit one single modality for all diagnostic applications. Therefore, the need for a multi or hybrid modality imaging arises. High-resolution imaging with optimal working distance, imaging around opaque obstacles are also of prime important in today's imaging world. However, the quest for multi-modality settings for the diagnostic imaging has posed subduing effects of certain advantages of the respective individual modalities. From these perspectives, a paradigm shift in medical diagnostics was introduced in the recent past by way of enhancing different parameters of interest using nanoscale contrast agents. A detailed analysis on the proposed schemes that can be explored based on the recent works carried out by the author's group for early diagnosis of cancerous growth in colon, and ocular imaging targeting iridocorneal angle and imaging of cornea will be reviewed from this perspective. A high resolution probe with selective spatiotemporal imaging that can find potential applications in opto-genetics will also be discussed.

Author acknowledges the financial support received through School of MAE, NTU and the Ministry of Education (MOE), Singapore through research project (RG 162/15). 